\title{
On the strong convergence for weighted sums of negatively superadditive dependent random variables
}

Bing Meng ${ }^{1,2}$, Dingcheng Wang ${ }^{1 *}$ and Qunying $W^{2}$

"Correspondence:
wangdc@uestc.edu.cn
'School of Mathematical Science,
University of Electronic Science and
Technology of China, Chengdu,
Sichuan 611731, P.R. China
Full list of author information is
available at the end of the article

available at the end of the article

\begin{abstract}
In this article, some strong convergence results for weighted sums of negatively superadditive dependent random variables are studied without assumption of identical distribution. The results not only generalize the corresponding ones of Cai (Metrika 68:323-331, 2008) and Sung (Stat. Pap. 52:447-454, 2011), but also extend and improve the corresponding one of Chen and Sung (Stat. Probab. Lett. 92:45-52, 2014).
\end{abstract}

MSC: $60 F 15$

Keywords: negatively superadditive dependent random variables; strong convergence; weighted sums

\section{Introduction}

Let $\left\{X_{n} ; n \geq 1\right\}$ be a sequence of random variables defined on a fixed probability space $(\Omega, \mathcal{F}, P)$. We first review the definitions of negatively associated random variables and negatively superadditive dependent (NSD) random variables.

Definition 1.1 A finite family of random variables $\left\{X_{i} ; 1 \leq i \leq n\right\}$ is said to be negatively associated (NA) if for every pair of disjoint subsets $A_{1}$ and $A_{2}$ of $\{1,2, \ldots, n\}$,

$$
\operatorname{Cov}\left(f_{1}\left(X_{i}, i \in A_{1}\right), f_{2}\left(X_{j}, j \in A_{2}\right)\right) \leq 0,
$$

whenever $f_{1}$ and $f_{2}$ are coordinatewise nondecreasing functions such that this covariance exists. An infinite family of random variables $\left\{X_{n} ; n \geq 1\right\}$ is said to be NA if every finite subfamily is NA.

Definition 1.2 (Kemperman [4]) A function $\phi: \mathbb{R}^{n} \rightarrow \mathbb{R}$ is called superadditive if $\phi(x \vee$ $y)+\phi(x \wedge y) \geq \phi(x)+\phi(y)$ for all $x, y \in \mathbb{R}^{n}$, where $\vee$ is for a componentwise maximum and $\wedge$ is for a componentwise minimum.

Definition $1.3(\mathrm{Hu}[5])$ A random vector $X=\left(X_{1}, X_{2}, \ldots, X_{n}\right)$ is said to be NSD if

$$
E \phi\left(X_{1}, X_{2}, \ldots, X_{n}\right) \leq E \phi\left(X_{1}^{*}, X_{2}^{*}, \ldots, X_{n}^{*}\right)
$$

(c) The Author(s) 2017. This article is distributed under the terms of the Creative Commons Attribution 4.0 International License (http://creativecommons.org/licenses/by/4.0/), which permits unrestricted use, distribution, and reproduction in any medium, provided you give appropriate credit to the original author(s) and the source, provide a link to the Creative Commons license, and indicate if changes were made. 
where $X_{1}^{*}, X_{2}^{*}, \ldots, X_{n}^{*}$ are independent such that $X_{i}^{*}$ and $X_{i}$ have the same distribution for each $i$ and $\phi$ is a superadditive function such that the expectations in (1.2) exist. A sequence of random variables $\left\{X_{n} ; n \geq 1\right\}$ is said to be NSD if for every $n \geq 1,\left(X_{1}, X_{2}, \ldots, X_{n}\right)$ is NSD.

The concept of NA was given by Joag-Dev and Proschan [6], and the concept of NSD was introduced by $\mathrm{Hu}$ [5], which was based on the class of superadditive functions. $\mathrm{Hu}$ [5] gave an example illustrating that NSD random variables are not necessarily NA, and left an open problem whether NA random variables implies NSD. Christofides and Vaggelatou [7] solved this open problem and showed that NA implies NSD. Thus, it is shown that NSD is much weaker than NA. Because of the wide application of NSD random variables, many authors have studied this concept and obtained some interesting results and applications. For example, we refer to [8-13]. Hence, it is of important significance to extend the limit properties of NA to the case of NSD random variables.

The concept of complete convergence was introduced by Hsu and Robbins [14] as follows. A sequence of random variables $\left\{X_{n} ; n \geq 1\right\}$ is said to converge completely to a con$\operatorname{stant} \lambda$ if

$$
\sum_{n=1}^{\infty} P\left(\left|X_{n}-\lambda\right|>\varepsilon\right)<\infty \quad \text { for all } \varepsilon>0
$$

In view of the Borel-Cantelli lemma, the sequence of random variables $\left\{X_{n} ; n \geq 1\right\}$ converging completely to a constant $\lambda$ implies $X_{n} \rightarrow \lambda$ almost surely (a.s.). Therefore, the complete convergence of random variables is a very important tool in establishing almost sure convergence. The first results concerning complete convergence for normed sums of random variables were due to Hsu and Robbins (1947) [14] and Erdös (1949) [15], and the obtained results have been extended in several directions by many authors. One can refer to [16-20], etc.

Recently, Cai [1] obtained the following complete convergence result for weighted sums of NA random variables with identical distribution.

Theorem 1.1 Let $\left\{X, X_{n} ; n \geq 1\right\}$ be a sequence of NA random variables with identical distribution, and let $\left\{a_{n i}, 1 \leq i \leq n, n \geq 1\right\}$ be a triangular array of constants satisfying $\sum_{i=1}^{n}\left|a_{n i}\right|^{\alpha}=O(n)$ for some $0<\alpha \leq 2$. Let $b_{n}=n^{1 / \alpha}(\log n)^{1 / \gamma}$ for some $\gamma>0$. Furthermore, assume that $E X=0$ when $1<\alpha \leq 2$. Iffor some $h>0$,

$$
E \exp \left(h|X|^{\gamma}\right)<\infty
$$

then, for all $\varepsilon>0$,

$$
\sum_{n=1}^{\infty} \frac{1}{n} P\left(\max _{1 \leq j \leq n}\left|\sum_{i=1}^{j} a_{n i} X_{i}\right|>\varepsilon b_{n}\right)<\infty
$$

Sung [2] extended the result of Cai [1] under a much weaker moment condition and obtained the following strong convergence results.

Theorem 1.2 Let $\left\{X, X_{n} ; n \geq 1\right\}$ be a sequence of $N A$ random variables with identical distribution, and let $\left\{a_{n i}, 1 \leq i \leq n, n \geq 1\right\}$ be an array of constants such that $\sum_{i=1}^{n}\left|a_{n i}\right|^{\alpha}=O(n)$ 
for some $0<\alpha \leq 2$. Let $b_{n}=n^{1 / \alpha}(\log n)^{1 / \gamma}$ for some $\gamma>0$. Furthermore, suppose that $E X=0$ when $1<\alpha \leq 2$. Then:

(i) If $\alpha>\gamma$, then $E|X|^{\alpha}<\infty$ implies (1.5).

(ii) If $\alpha=\gamma$, then $E|X|^{\alpha} \log (1+|X|)<\infty$ implies (1.5).

(iii) If $\alpha<\gamma$, then $E|X|^{\gamma}<\infty$ implies (1.5).

In the case $\alpha>\gamma$, Chen and Sung [3] studied the complete convergence for weighted sums of NA random variables under the moment condition $E|X|^{\alpha} /(\log (1+|X|))^{\alpha / \gamma-1}<\infty$, which is weaker than Theorem 1.2. Li et al. [21] extended and improved the result of Chen and Sung [3] to $\rho^{*}$-mixing random variables. Motivated by the above results obtained by Cai [1], Sung [2] and Chen and Sung [3], in this paper, we will further study the complete convergence for weighted sums of NSD random variables. Some complete convergence results for the maximum weighted sums of NSD random variables are obtained without the assumption of an identical distribution. As an application, the Marcinkiewicz-Zygmund type strong law of large numbers for weighted sums of NSD random variables is obtained. Our results not only generalize the corresponding ones of Cai [1] and Sung [2], but they also extend and improve the corresponding one of Chen and Sung [3].

\section{Preliminaries}

Throughout this paper, $C$ represents a generic positive constant whose value may change from one appearance to the next, and $a_{n}=O\left(b_{n}\right)$ means $a_{n} \leq C b_{n}$. Let $I(A)$ be the indicator function of the set $A$.

Definition 2.1 A sequence of random variables $\left\{X_{n} ; n \geq 1\right\}$ is said to be stochastically dominated by a random variable $X$ if there exists a positive constant $C$ such that

$$
P\left(\left|X_{n}\right| \geq x\right) \leq C P(|X| \geq x)
$$

for all $x \geq 0$ and $n \geq 1$.

In order to prove our main results, we introduce the following lemmas.

Lemma 2.1 $(\mathrm{Hu}[5])$ If $X=\left(X_{1}, X_{2}, \ldots, X_{n}\right)$ is $N S D$ and $f_{1}, f_{2}, \ldots, f_{n}$ are nondecreasing functions, then $\left(f_{1}\left(X_{1}\right), f_{2}\left(X_{2}\right), \ldots, f_{n}\left(X_{n}\right)\right)$ is NSD.

Lemma 2.2 (Wang et al. [11]) Let $p>1$ and $\left\{X_{n} ; n \geq 1\right\}$ be a sequence of NSD random variables with $E\left|X_{i}\right|^{p}<\infty$ for every $i \geq 1$. Then, there exists a positive constant $C=C_{p}$ depending only on $p$ such that, for every $n \geq 1$, for $1<p \leq 2$,

$$
E\left(\max _{1 \leq j \leq n}\left|\sum_{i=1}^{j} X_{i}\right|^{p}\right) \leq C \sum_{i=1}^{n} E\left|X_{i}\right|^{p}
$$

and, for $p>2$,

$$
E\left(\max _{1 \leq j \leq n}\left|\sum_{i=1}^{j} X_{i}\right|^{p}\right) \leq C\left\{\sum_{i=1}^{n} E\left|X_{i}\right|^{p}+\left(\sum_{i=1}^{n} E X_{i}^{2}\right)^{p / 2}\right\} .
$$


Lemma 2.3 (Sung [22]) Let $X$ be a random variable and $\left\{a_{n i} ; 1 \leq i \leq n, n \geq 1\right\}$ be an array of constants satisfying $\sum_{i=1}^{n}\left|a_{n i}\right|^{\alpha}=O(n)$ for some $\alpha>0$. Let $b_{n}=n^{1 / \alpha}(\log n)^{1 / \gamma}$ for some $\gamma>0$. Then

$$
\sum_{n=1}^{\infty} \frac{1}{n} \sum_{i=1}^{n} P\left(\left|a_{n i} X\right|>b_{n}\right) \leq \begin{cases}C E|X|^{\alpha}, & \text { for } \alpha>\gamma \\ C E|X|^{\alpha} \log (1+|X|), & \text { for } \alpha=\gamma \\ C E|X|^{\gamma}, & \text { for } \alpha<\gamma .\end{cases}
$$

Lemma 2.4 (Sung [22]) Let $X$ be a random variable and $\left\{a_{n i} ; 1 \leq i \leq n, n \geq 1\right\}$ be an array of constants satisfying $a_{n i}=0$ or $\left|a_{n i}\right|>1$, and $\sum_{i=1}^{n}\left|a_{n i}\right|^{\alpha}=O(n)$ for some $\alpha>0$. Let $b_{n}=$ $n^{1 / \alpha}(\log n)^{1 / \alpha}$. If $p>\alpha$, then

$$
\sum_{n=1}^{\infty} n^{-1} b_{n}^{-p} \sum_{i=1}^{n} E\left|a_{n i} X\right|^{p} I\left(\left|a_{n i} X\right| \leq b_{n}\right) \leq C E|X|^{\alpha} \log (1+|X|) .
$$

Lemma 2.5 (Wu [23]) Let $\left\{X_{n} ; n \geq 1\right\}$ be a sequence of random variables which is stochastically dominated by a random variable $X$. For any $u>0, t>0$ and $n \geq 1$, the following two statements hold:

$$
\begin{aligned}
& E\left|X_{n}\right|^{u} I\left(\left|X_{n}\right| \leq t\right) \leq C\left[E|X|^{u} I(|X| \leq t)+t^{u} P(|X|>t)\right], \\
& E\left|X_{n}\right|^{u} I\left(\left|X_{n}\right|>t\right) \leq C E|X|^{u} I(|X|>t) .
\end{aligned}
$$

\section{Main results and proofs}

Now we state and prove our main results.

Theorem 3.1 Let $\left\{X_{n} ; n \geq 1\right\}$ be a sequence of NSD random variables which is stochastically dominated by a random variable $X$, and $b_{n}=n^{1 / \alpha}(\log n)^{1 / \gamma}$ for some $0<\alpha \leq 2$ and $\gamma>0$. Let $\left\{a_{n i} ; 1 \leq i \leq n, n \geq 1\right\}$ be an array of constants satisfying $\sum_{i=1}^{n}\left|a_{n i}\right|^{\gamma}=O(n)$. Assume further that $E X_{n}=0$ when $1<\alpha \leq 2$. Then:

(i) If $\alpha<\gamma$, then $E|X|^{\gamma}<\infty$ implies (1.5).

(ii) If $\alpha=\gamma$, then $E|X|^{\alpha} \log (1+|X|)<\infty$ implies (1.5).

Theorem 3.2 Let $\left\{X_{n} ; n \geq 1\right\}$ be a sequence of NSD random variables which is stochastically dominated by a random variable $X$, and $b_{n}=n^{1 / \alpha}(\log n)^{1 / \gamma}$ for some $0<\alpha \leq 2$ and $\gamma>0$. Let $\left\{a_{n i} ; 1 \leq i \leq n, n \geq 1\right\}$ be an array of constants satisfying $\sum_{i=1}^{n}\left|a_{n i}\right|^{\alpha}=O(n)$. Assume further that $E X_{n}=0$ when $1<\alpha \leq 2$. If $\alpha>\gamma$, then $E|X|^{\alpha} /(\log (1+|X|))^{\alpha / \gamma-1}<\infty$ implies (1.5).

Remark 3.1 In Theorem 3.1 and Theorem 3.2, we use different methods from those of Sung [2] and Chen and Sung [3] to prove the results, and obtain some strong convergence results for weighted sums of NSD random variables without assumptions of identical distribution. The obtained theorems not only extend the corresponding results of Cai [1] and Sung [2] and Chen and Sung [3] to the case of NSD random variables, but they also improve them. 
Proof of Theorem 3.1 Without loss of generality, we suppose that $a_{n i}>0$. For $\forall i \geq 1$, define

$$
\begin{aligned}
& Y_{i}=-b_{n} I\left(a_{n i} X_{n i}<-b_{n}\right)+a_{n i} X_{n i} I\left(\left|a_{n i} X_{n i}\right| \leq b_{n}\right)+b_{n} I\left(a_{n i} X_{n i}>b_{n}\right), \\
& T_{j}=\sum_{i=1}^{j}\left(Y_{i}-E Y_{i}\right), \quad j=1,2, \ldots, n
\end{aligned}
$$

It is easy to check that, for all $\varepsilon>0$,

$$
\left\{\max _{1 \leq j \leq n}\left|\sum_{i=1}^{j} a_{n i} X_{i}\right|>\varepsilon b_{n}\right\} \subset\left\{\max _{1 \leq j \leq n}\left|a_{n j} X_{j}\right|>b_{n}\right\} \cup\left\{\max _{1 \leq j \leq n}\left|\sum_{i=1}^{j} Y_{i}\right|>\varepsilon b_{n}\right\},
$$

which implies that

$$
\begin{aligned}
& P\left(\max _{1 \leq j \leq n}\left|\sum_{i=1}^{j} a_{n i} X_{i}\right|>\varepsilon b_{n}\right) \\
& \quad \leq P\left(\max _{1 \leq j \leq n}\left|\sum_{i=1}^{j} Y_{i}\right|>\varepsilon b_{n}\right)+P\left(\max _{1 \leq j \leq n}\left|a_{n j} X_{j}\right|>b_{n}\right) \\
& \quad \leq P\left(\max _{1 \leq j \leq n}\left|T_{j}\right|>\varepsilon b_{n}-\max _{1 \leq j \leq n}\left|\sum_{i=1}^{j} E Y_{i}\right|\right)+\sum_{j=1}^{n} P\left(\left|a_{n j} X_{j}\right|>b_{n}\right) .
\end{aligned}
$$

Firstly, we prove that

$$
b_{n}^{-1} \max _{1 \leq j \leq n}\left|\sum_{i=1}^{j} E Y_{i}\right| \rightarrow 0, \quad \text { as } n \rightarrow \infty
$$

If $1<\alpha \leq 2$, then by $E X_{n}=0$, Lemma 2.5, Definition 2.1, the $C_{r}$ inequality, the Markov inequality and the Hölder inequality, we get

$$
\begin{aligned}
b_{n}^{-1} \max _{1 \leq j \leq n}\left|\sum_{i=1}^{j} E Y_{i}\right| & \leq b_{n}^{-1} \sum_{i=1}^{n}\left|E Y_{i}\right| \\
& \leq b_{n}^{-1} \sum_{i=1}^{n}\left|E a_{n i} X_{i} I\left(\left|a_{n i} X_{i}\right| \leq b_{n}\right)\right|+\sum_{i=1}^{n} P\left(\left|a_{n i} X_{i}\right|>b_{n}\right) \\
& \leq C b_{n}^{-1} \sum_{i=1}^{n} E\left|a_{n i} X\right| I\left(\left|a_{n i} X\right|>b_{n}\right)+C \sum_{i=1}^{n} P\left(\left|a_{n i} X\right|>b_{n}\right) \\
& \leq C b_{n}^{-\alpha} \sum_{i=1}^{n} E\left|a_{n i} X\right|^{\alpha} I\left(\left|a_{n i} X\right|>b_{n}\right)+C b_{n}^{-\alpha} \sum_{i=1}^{n} E\left|a_{n i} X\right|^{\alpha} \\
& \leq C b_{n}^{-\alpha} \sum_{i=1}^{n} E\left|a_{n i} X\right|^{\alpha}+C b_{n}^{-\alpha} \sum_{i=1}^{n} E\left|a_{n i} X\right|^{\alpha} \\
& \leq C b_{n}^{-\alpha} \sum_{i=1}^{n}\left|a_{n i}\right|^{\gamma} E|X|^{\alpha} \\
& \leq C(\log n)^{-\alpha / \gamma} \rightarrow 0, \quad \text { as } n \rightarrow \infty .
\end{aligned}
$$


If $0<\alpha \leq 1$, then by Lemma 2.5, Definition 2.1, the $C_{r}$ inequality and the Markov inequality, we get again

$$
\begin{aligned}
b_{n}^{-1} \max _{1 \leq j \leq n}\left|\sum_{i=1}^{j} E Y_{i}\right| \leq & b_{n}^{-1} \sum_{i=1}^{n}\left|E Y_{i}\right| \\
\leq & b_{n}^{-1} \sum_{i=1}^{n}\left|E a_{n i} X_{i} I\left(\left|a_{n i} X_{i}\right| \leq b_{n}\right)\right|+\sum_{i=1}^{n} P\left(\left|a_{n i} X_{i}\right|>b_{n}\right) \\
\leq & C b_{n}^{-1} \sum_{i=1}^{n}\left[E\left|a_{n i} X\right| I\left(\left|a_{n i} X\right| \leq b_{n}\right)+b_{n} P\left(\left|a_{n i} X\right|>b_{n}\right)\right] \\
& +C \sum_{i=1}^{n} P\left(\left|a_{n i} X\right|>b_{n}\right) \\
\leq & C b_{n}^{-\alpha} \sum_{i=1}^{n} E\left|a_{n i} X\right|^{\alpha}+C b_{n}^{-\alpha} \sum_{i=1}^{n} E\left|a_{n i} X\right|^{\alpha} \\
\leq & C b_{n}^{-\alpha} \sum_{i=1}^{n}\left|a_{n i}\right|^{\gamma} E|X|^{\alpha} \\
\leq & C(\log n)^{-\alpha / \gamma} \rightarrow 0, \quad \text { as } n \rightarrow \infty .
\end{aligned}
$$

It immediately follows from (3.4) and (3.5), that (3.3) holds. Hence, for $n$ large enough,

$$
P\left(\max _{1 \leq j \leq n}\left|\sum_{i=1}^{j} a_{n i} X_{i}\right|>\varepsilon b_{n}\right) \leq \sum_{i=1}^{n} P\left(\left|a_{n i} X_{i}\right|>b_{n}\right)+P\left(\max _{1 \leq j \leq n}\left|T_{j}>\frac{\varepsilon b_{n}}{2}\right|\right) .
$$

Then, to prove (1.5), it suffices to prove that

$$
I \triangleq \sum_{n=1}^{\infty} \frac{1}{n} \sum_{i=1}^{n} P\left(\left|a_{n i} X_{i}\right|>b_{n}\right)<\infty
$$

and

$$
J \triangleq \sum_{n=1}^{\infty} \frac{1}{n} P\left(\max _{1 \leq j \leq n}\left|T_{j}\right|>\frac{\varepsilon b_{n}}{2}\right)<\infty
$$

By Lemma 2.3, we can easily obtain

$$
\begin{aligned}
I & \triangleq \sum_{n=1}^{\infty} \frac{1}{n} \sum_{i=1}^{n} P\left(\left|a_{n i} X_{i}\right|>b_{n}\right) \\
& \leq C \sum_{n=1}^{\infty} \frac{1}{n} \sum_{i=1}^{n} P\left(\left|a_{n i} X\right|>b_{n}\right)<\infty .
\end{aligned}
$$

For fixed $n \geq 1$, it is easily seen that $\left\{Y_{i} ; 1 \leq i \leq n\right\}$ is still a sequence of NSD random variables by Lemma 2.1. Hence, for $p>2$, it follows from Lemma 2.2, the Markov inequality 
and the Jensen inequality that

$$
\begin{aligned}
J & \triangleq \sum_{n=1}^{\infty} \frac{1}{n} P\left(\max _{1 \leq j \leq n}\left|T_{j}\right|>\frac{\varepsilon b_{n}}{2}\right) \\
& \leq C \sum_{n=1}^{\infty} \frac{1}{n} b_{n}^{-p} E\left(\max _{1 \leq j \leq n}\left|T_{j}\right|^{p}\right) \\
& \leq C \sum_{n=1}^{\infty} n^{-1} b_{n}^{-p}\left(\sum_{i=1}^{n} E\left|Y_{i}-E Y_{i}\right|^{p}+\left(\sum_{i=1}^{n} E\left|Y_{i}-E Y_{i}\right|^{2}\right)^{p / 2}\right) \\
& \leq C \sum_{n=1}^{\infty} n^{-1} b_{n}^{-p} \sum_{i=1}^{n} E\left|Y_{i}\right|^{p}+C \sum_{n=1}^{\infty} n^{-1} b_{n}^{-p}\left(\sum_{i=1}^{n} E\left|Y_{i}\right|^{2}\right)^{p / 2} \\
& \triangleq J_{1}+J_{2} .
\end{aligned}
$$

Firstly, we prove $J_{1}<\infty$. By Lemma 2.3 , we obtain

$$
\begin{aligned}
J_{1} & \triangleq C \sum_{n=1}^{\infty} n^{-1} b_{n}^{-p} \sum_{i=1}^{n} E\left|Y_{i}\right|^{p} \\
& \leq C \sum_{n=1}^{\infty} n^{-1} b_{n}^{-p} \sum_{i=1}^{n}\left[E\left|a_{n i} X\right|^{p} I\left(\left|a_{n i} X\right| \leq b_{n}\right)+b_{n}^{p} P\left(\left|a_{n i} X\right|>b_{n}\right)\right] \\
& =C \sum_{n=1}^{\infty} n^{-1} b_{n}^{-p} \sum_{i=1}^{n} E\left|a_{n i} X\right|^{p} I\left(\left|a_{n i} X\right| \leq b_{n}\right)+C \sum_{n=1}^{\infty} n^{-1} \sum_{i=1}^{n} P\left(\left|a_{n i} X\right|>b_{n}\right) \\
& \triangleq J_{11}+J_{12} .
\end{aligned}
$$

Actually, by Lemma 2.3 , we can directly obtain $J_{12}<\infty$. Hence, we only need to prove $J_{11}<\infty$ in the following two cases.

(i) If $\alpha<\gamma$, take $p>\max \{2, \gamma\}$, then by $\sum_{i=1}^{n}\left|a_{n i}\right|^{\gamma} \leq C n$ and $E|X|^{\gamma}<\infty$ it follows that

$$
\begin{aligned}
J_{11} & =C \sum_{n=1}^{\infty} n^{-1} b_{n}^{-p} \sum_{i=1}^{n} E\left|a_{n i} X\right|^{p} I\left(\left|a_{n i} X\right| \leq b_{n}\right) \\
& =C \sum_{n=1}^{\infty} n^{-1} \sum_{i=1}^{n} E\left|\frac{a_{n i} X}{b_{n}}\right|^{p} I\left(\left|a_{n i} X\right| \leq b_{n}\right) \\
& \leq C \sum_{n=1}^{\infty} n^{-1} \sum_{i=1}^{n} E\left|\frac{a_{n i} X}{b_{n}}\right|^{\gamma} I\left(\left|a_{n i} X\right| \leq b_{n}\right) \\
& \leq C \sum_{n=1}^{\infty} n^{-1} b_{n}^{-\gamma} \sum_{i=1}^{n} E\left|a_{n i} X\right|^{\gamma} I\left(\left|a_{n i} X\right| \leq b_{n}\right) \\
& \leq C \sum_{n=1}^{\infty} n^{-1} b_{n}^{-\gamma} \sum_{i=1}^{n}\left|a_{n i}\right|^{\gamma} E|X|^{\gamma} \\
& \leq C \sum_{n=1}^{\infty} n^{-1} n^{-\gamma / \alpha}(\log n)^{-1} n E|X|^{\gamma} \\
& \leq C \sum_{n=1}^{\infty} n^{-\gamma / \alpha}(\log n)^{-1}<\infty .
\end{aligned}
$$


(ii) If $\alpha=\gamma$, we need to divide $\left\{a_{n i} ; 1 \leq i \leq n, n \geq 1\right\}$ into three subsets: $\left\{a_{n i}:\left|a_{n i}\right| \leq\right.$ $\left.1 /(\log n)^{t}\right\},\left\{a_{n i}: 1 /(\log n)^{t}<\left|a_{n i}\right| \leq 1\right\}$ and $\left\{a_{n i}:\left|a_{n i}\right|>1\right\}$, where $t=1 /(p-\alpha)$. Then we obtain

$$
\begin{aligned}
J_{11}= & C \sum_{n=1}^{\infty} n^{-1} b_{n}^{-p} \sum_{i=1}^{n} E\left|a_{n i} X\right|^{p} I\left(\left|a_{n i} X\right| \leq b_{n}\right) \\
= & C \sum_{n=1}^{\infty} n^{-1} b_{n}^{-p} \sum_{i:\left|a_{n i}\right| \leq 1 /(\log n)^{t}}^{n} E\left|a_{n i} X\right|^{p} I\left(\left|a_{n i} X\right| \leq b_{n}\right) \\
& +C \sum_{n=1}^{\infty} n^{-1} b_{n}^{-p} \sum_{i: 1 /(\log n)^{t}<\left|a_{n i}\right| \leq 1}^{n} E\left|a_{n i} X\right|^{p} I\left(\left|a_{n i} X\right| \leq b_{n}\right) \\
& +C \sum_{n=1}^{\infty} n^{-1} b_{n}^{-p} \sum_{i:\left|a_{n i}\right|>1}^{n} E\left|a_{n i} X\right|^{p} I\left(\left|a_{n i} X\right| \leq b_{n}\right) \\
\triangleq & J_{111}+J_{112}+J_{113} .
\end{aligned}
$$

Obviously, by Lemma 2.4 , we directly obtain $J_{113} \leq E|X|^{\alpha} \log (1+|X|)<\infty$.

It follows from $\sum_{i:\left|a_{n i}\right| \leq 1 /(\log n)^{t}}\left|a_{n i}\right|^{\alpha} \leq C n(\log n)^{-t \alpha}$ and $E|X|^{\alpha}<\infty$ that

$$
\begin{aligned}
J_{111} & =C \sum_{n=1}^{\infty} n^{-1} b_{n}^{-p} \sum_{i:\left|a_{n i}\right| \leq 1 /(\log n)^{t}}^{n} E\left|a_{n i} X\right|^{p} I\left(\left|a_{n i} X\right| \leq b_{n}\right) \\
& \leq C \sum_{n=1}^{\infty} n^{-1} b_{n}^{-p} \sum_{i:\left|a_{n i}\right| \leq 1 /(\log n)^{t}}^{n}\left|a_{n i}\right|^{p} E|X|^{p} I\left(\left|a_{n i} X\right| \leq b_{n}\right) \\
& \leq C \sum_{n=1}^{\infty} n^{-1} b_{n}^{-\alpha} \sum_{i:\left|a_{n i}\right| \leq 1 /(\log n)^{t}}\left|a_{n i}\right|^{\alpha} E|X|^{\alpha} I\left(\left|a_{n i} X\right| \leq b_{n}\right) \\
& \leq C \sum_{n=1}^{\infty} n^{-1} b_{n}^{-\alpha} E|X|^{\alpha} \sum_{i:\left|a_{n i}\right| \leq 1 /(\log n)^{t}}^{n}\left|a_{n i}\right|^{\alpha} \\
& \leq C \sum_{n=1}^{\infty} n^{-1} b_{n}^{-\alpha} E|X|^{\alpha} \sum_{i:\left|a_{n i}\right| \leq 1 /(\log n)^{t}}^{n}\left|a_{n i}\right|^{\alpha} \\
& \leq C \sum_{n=1}^{\infty} E|X|^{\alpha} n^{-1}(\log n)^{-1-t \alpha} \\
& \leq C \sum_{n=1}^{\infty} n^{-1}(\log n)^{-1-t \alpha}<\infty .
\end{aligned}
$$

It follows from $\sum_{i: 1 /(\log n)^{t}<\left|a_{n i}\right| \leq 1}\left|a_{n i}\right|^{p} \leq C n, E|X|^{\alpha}<\infty$ and $t=1 /(p-\alpha)$ for $p>2,0<\alpha \leq 2$ that

$$
\begin{aligned}
J_{112} & =C \sum_{n=1}^{\infty} n^{-1} b_{n}^{-p} \sum_{i: 1 /(\log n)^{t}<\left|a_{n i}\right| \leq 1}^{n} E\left|a_{n i} X\right|^{p} I\left(\left|a_{n i} X\right| \leq b_{n}\right) \\
& \leq C \sum_{n=1}^{\infty} n^{-1} b_{n}^{-p} \sum_{i: 1 /(\log n)^{t}<\left|a_{n i}\right| \leq 1}^{n}\left|a_{n i}\right|^{p} E|X|^{p} I\left(\left|a_{n i} X\right| \leq b_{n}\right)
\end{aligned}
$$




$$
\begin{aligned}
\leq & C \sum_{n=1}^{\infty} b_{n}^{-p} E|X|^{p} I\left(|X| \leq b_{n}(\log n)^{t}\right) \\
\leq & C \sum_{n=1}^{\infty} b_{n}^{-p} \sum_{k=1}^{n} E|X|^{p} I\left((k-1)^{1 / \alpha}(\log (k-1))^{t+1 / \alpha}<|X| \leq k^{1 / \alpha}(\log k)^{t+1 / \alpha}\right) \\
\leq & C \sum_{k=1}^{\infty} E|X|^{p} I\left((k-1)^{1 / \alpha}(\log (k-1))^{t+1 / \alpha}<|X| \leq k^{1 / \alpha}(\log k)^{t+1 / \alpha}\right) \\
& \times \sum_{n=k}^{\infty} n^{-p / \alpha}(\log n)^{-p / \alpha} \\
\leq & C \sum_{k=1}^{\infty} E|X|^{\alpha} \frac{k^{p / \alpha}(\log k)^{p(t+1 / \alpha)}}{(k-1)(\log (k-1))^{\alpha t+1}} k^{1-p / \alpha}(\log k)^{-p / \alpha} \\
\leq & C E|X|^{\alpha}<\infty .
\end{aligned}
$$

Therefore, by (3.11)-(3.15), we can see that $J_{1}<\infty$. Finally, we prove $J_{2}<\infty$. Actually, take $p>\max \left\{2, \frac{2 \gamma}{\alpha}\right\}$, then by Lemma 2.5 , the Markov inequality and $E|X|^{\gamma}<\infty$, we get

$$
\begin{aligned}
J_{2}= & C \sum_{n=1}^{\infty} n^{-1} b_{n}^{-p}\left(\sum_{i=1}^{n} E\left|Y_{i}\right|^{2}\right)^{p / 2} \\
\leq & C \sum_{n=1}^{\infty} n^{-1} b_{n}^{-p}\left(\sum_{i=1}^{n} E\left|a_{n i} X\right|^{2} I\left(\left|a_{n i} X_{i}\right| \leq b_{n}\right)\right)^{p / 2} \\
& +C \sum_{n=1}^{\infty} n^{-1} b_{n}^{-p}\left(\sum_{i=1}^{n} b_{n}^{2} P\left(\left|a_{n i} X_{i}\right|>b_{n}\right)\right)^{p / 2} \\
\leq & C \sum_{n=1}^{\infty} n^{-1} b_{n}^{-p}\left(\sum_{i=1}^{n}\left[E\left|a_{n i} X\right|^{2} I\left(\left|a_{n i} X\right| \leq b_{n}\right)+b_{n}^{2} P\left(\left|a_{n i} X\right|>b_{n}\right)\right]\right)^{p / 2} \\
& +C \sum_{n=1}^{\infty} n^{-1}\left(\sum_{i=1}^{n} P\left(\left|a_{n i} X\right|>b_{n}\right)\right)^{p / 2} \\
\leq & C \sum_{n=1}^{\infty} n^{-1}\left(\sum_{i=1}^{n} b_{n}^{-2} E\left|a_{n i} X\right|^{2} I\left(\left|a_{n i} X\right| \leq b_{n}\right)\right)^{p / 2} \\
& +C \sum_{n=1}^{\infty} n^{-1}\left(\sum_{i=1}^{n} P\left(\left|a_{n i} X\right|>b_{n}\right)\right)^{p / 2} \\
\leq & C \sum_{n=1}^{\infty} n^{-1}\left(\sum_{i=1}^{n} b_{n}^{-\alpha} E\left|a_{n i} X\right|^{\alpha} I\left(\left|a_{n i} X\right| \leq b_{n}\right)\right)^{p / 2} \\
& +C \sum_{n=1}^{\infty} n^{-1}\left(\sum_{i=1}^{n} b_{n}^{-\alpha} E\left|a_{n i} X\right|^{\alpha} n^{-1} b_{n}^{-\alpha p / 2}\left(\sum_{i=1}^{n}\left|a_{n i}\right|^{\alpha} E|X|^{\alpha}\right)^{p / 2}\right. \\
&
\end{aligned}
$$




$$
\begin{aligned}
& \leq C \sum_{n=1}^{\infty} n^{-1} b_{n}^{-\alpha p / 2} n^{p / 2} \\
& =C \sum_{n=1}^{\infty} n^{-1} n^{-p / 2}(\log n)^{-\frac{\alpha p}{2 \gamma}} n^{p / 2} \\
& =C \sum_{n=1}^{\infty} n^{-1}(\log n)^{-\frac{\alpha p}{2 \gamma}}<\infty .
\end{aligned}
$$

Thus, the proof of Theorem 3.1 is completed.

Proof of Theorem 3.2 Without loss of generality, we suppose that $a_{n i}>0$. For $\forall i \geq 1$, define

$$
Z_{i}=a_{n i} X_{n i} I\left(\left|a_{n i} X_{n i}\right| \leq b_{n}\right)
$$

It is easy to check that, for all $\varepsilon>0$,

$$
\left\{\max _{1 \leq j \leq n}\left|\sum_{i=1}^{j} a_{n i} X_{i}\right|>\varepsilon b_{n}\right\} \subset\left\{\max _{1 \leq j \leq n}\left|a_{n j} X_{j}\right|>b_{n}\right\} \cup\left\{\max _{1 \leq j \leq n}\left|\sum_{i=1}^{j} Z_{i}\right|>\varepsilon b_{n}\right\},
$$

which implies that

$$
\begin{aligned}
& P\left(\max _{1 \leq j \leq n}\left|\sum_{i=1}^{j} a_{n i} X_{i}\right|>\varepsilon b_{n}\right) \\
& \quad \leq P\left(\max _{1 \leq j \leq n}\left|\sum_{i=1}^{j} Z_{i}\right|>\varepsilon b_{n}\right)+P\left(\max _{1 \leq j \leq n}\left|a_{n j} X_{j}\right|>b_{n}\right) \\
& \quad \leq P\left(\max _{1 \leq j \leq n}\left|\sum_{i=1}^{j} Z_{i}\right|>\varepsilon b_{n}\right)+\sum_{j=1}^{n} P\left(\left|a_{n j} X_{j}\right|>b_{n}\right) .
\end{aligned}
$$

To prove (1.5), it suffices to show that

$$
H \triangleq \sum_{n=1}^{\infty} \frac{1}{n} \sum_{i=1}^{n} P\left(\left|a_{n i} X_{i}\right|>b_{n}\right)<\infty
$$

and

$$
L \triangleq \sum_{n=1}^{\infty} \frac{1}{n} P\left(\max _{1 \leq j \leq n}\left|\sum_{i=1}^{j} Z_{i}\right|>\varepsilon b_{n}\right)<\infty .
$$

We first prove (3.18). Note that

$$
P\left(\left|a_{n i} X_{i}\right|>b_{n}\right)=P\left(\left|a_{n i} X_{i}\right|>b_{n},\left|X_{i}\right|>b_{n}\right)+P\left(\left|a_{n i} X_{i}\right|>b_{n},\left|X_{i}\right| \leq b_{n}\right) .
$$

By the Markov inequality, we get

$$
P\left(\left|a_{n i} X_{i}\right|>b_{n},\left|X_{i}\right|>b_{n}\right) \leq b_{n}^{-\theta}\left|a_{n i}\right|^{\theta} E\left|X_{i}\right|^{\theta} I\left(\left|X_{i}\right|>b_{n}\right)
$$


for any $0<\theta<\alpha$ and

$$
P\left(\left|a_{n i} X_{i}\right|>b_{n},\left|X_{i}\right| \leq b_{n}\right) \leq b_{n}^{-\alpha}\left|a_{n i}\right|^{\alpha} E\left|X_{i}\right|^{\alpha} I\left(\left|X_{i}\right| \leq b_{n}\right) .
$$

It is easy to show that

$$
\begin{gathered}
\sum_{n=1}^{\infty} n^{-1} b_{n}^{-\theta} \sum_{i=1}^{n}\left|a_{n i}\right|^{\theta} E\left|X_{i}\right|^{\theta} I\left(\left|X_{i}\right|>b_{n}\right) \\
\leq C \sum_{n=1}^{\infty} b_{n}^{-\theta} E|X|^{\theta} I\left(|X|>b_{n}\right) \\
\leq C E|X|^{\alpha} /(\log (1+|X|))^{\alpha / \gamma}<\infty
\end{gathered}
$$

and

$$
\begin{aligned}
& \sum_{n=1}^{\infty} n^{-1} b_{n}^{-\alpha} \sum_{i=1}^{n}\left|a_{n i}\right|^{\alpha} E\left|X_{i}\right|^{\alpha} I\left(\left|X_{i}\right| \leq b_{n}\right) \\
& \quad \leq C \sum_{n=1}^{\infty} b_{n}^{-\alpha}\left[E|X|^{\alpha} I\left(|X| \leq b_{n}\right)+b_{n}^{\alpha} P\left(|X|>b_{n}\right)\right] \\
& \quad \leq C \sum_{n=1}^{\infty} b_{n}^{-\alpha} E|X|^{\alpha} I\left(|X| \leq b_{n}\right)+C \sum_{n=1}^{\infty} P\left(|X|>n^{1 / \alpha}(\log n)^{1 / \gamma}\right) \\
& \quad \leq C E|X|^{\alpha} /(\log (1+|X|))^{\alpha / \gamma-1}+C E|X|^{\alpha} /(\log (1+|X|))^{\alpha / \gamma}<\infty .
\end{aligned}
$$

Then, (3.18) holds by (3.20)-(3.24). Now we prove (3.19) in the following two cases.

(i) If $0<\alpha \leq 1$, similar to the proof of (3.18), we have

$$
\begin{aligned}
E\left|a_{n i} X_{i}\right|^{\alpha} I\left(\left|a_{n i} X_{i}\right| \leq b_{n}\right)= & E\left|a_{n i} X_{i}\right|^{\alpha} I\left(\left|a_{n i} X_{i}\right| \leq b_{n},\left|X_{i}\right|>b_{n}\right) \\
& +E\left|a_{n i} X_{i}\right|^{\alpha} I\left(\left|a_{n i} X_{i}\right| \leq b_{n},\left|X_{i}\right| \leq b_{n}\right) .
\end{aligned}
$$

Note that

$$
E\left|a_{n i} X_{i}\right|^{\alpha} I\left(\left|a_{n i} X_{i}\right| \leq b_{n},\left|X_{i}\right|>b_{n}\right) \leq b_{n}^{\alpha-\theta}\left|a_{n i}\right|^{\theta} E\left|X_{i}\right|^{\theta} I\left(\left|X_{i}\right|>b_{n}\right)
$$

for any $0<\theta<\alpha$ and

$$
E\left|a_{n i} X_{i}\right|^{\alpha} I\left(\left|a_{n i} X_{i}\right| \leq b_{n},\left|X_{i}\right| \leq b_{n}\right) \leq\left|a_{n i}\right|^{\alpha} E\left|X_{i}\right|^{\alpha} I\left(\left|X_{i}\right| \leq b_{n}\right) .
$$

By the Markov inequality, the $C_{r}$ inequality and (3.23)-(3.27), we obtain

$$
L \leq \sum_{n=1}^{\infty} n^{-1} b_{n}^{-\alpha} \sum_{i=1}^{n} E\left|a_{n i} X_{i}\right|^{\alpha} I\left(\left|a_{n i} X_{i}\right| \leq b_{n}\right)<\infty .
$$

(ii) If $1<\alpha \leq 2$, we first prove that

$$
b_{n}^{-1} \max _{1 \leq j \leq n}\left|\sum_{i=1}^{j} E a_{n i} X_{i} I\left(\left|a_{n i} X_{i}\right| \leq b_{n}\right)\right| \rightarrow 0, \quad \text { as } n \rightarrow \infty .
$$


By $E X_{n}=0$, we have

$$
\begin{aligned}
\max _{1 \leq j \leq n}\left|\sum_{i=1}^{j} E a_{n i} X_{i} I\left(\left|a_{n i} X_{i}\right| \leq b_{n}\right)\right| \leq & \sum_{i=1}^{n} E\left|a_{n i} X_{i}\right| I\left(\left|a_{n i} X_{i}\right|>b_{n}\right) \\
= & \sum_{i=1}^{n} E\left|a_{n i} X_{i}\right| I\left(\left|a_{n i} X_{i}\right|>b_{n},\left|X_{i}\right|>b_{n}\right) \\
& +\sum_{i=1}^{n} E\left|a_{n i} X_{i}\right| I\left(\left|a_{n i} X_{i}\right|>b_{n},\left|X_{i}\right| \leq b_{n}\right) .
\end{aligned}
$$

Since

$$
\begin{aligned}
& E\left|a_{n i} X_{i}\right| I\left(\left|a_{n i} X_{i}\right|>b_{n},\left|X_{i}\right|>b_{n}\right) \\
& \quad \leq\left|a_{n i}\right| E|X| I\left(|X|>b_{n}\right) \\
& \quad=\left|a_{n i}\right| E\left(\frac{|X|^{\alpha}}{(\log (1+|X|))^{\alpha / \gamma-1}} \cdot|X|^{1-\alpha}(\log (1+|X|))^{\alpha / \gamma-1}\right) I\left(|X|>b_{n}\right) \\
& \quad \leq C b_{n}^{1-\alpha}\left(\log \left(1+b_{n}\right)\right)^{\alpha / \gamma-1}\left|a_{n i}\right| \\
& \quad \leq C n^{-1+1 / \alpha}(\log n)^{1 / \gamma-1}\left|a_{n i}\right|
\end{aligned}
$$

and

$$
\begin{aligned}
E\left|a_{n i} X_{i}\right| I\left(\left|a_{n i} X_{i}\right|>b_{n},\left|X_{i}\right| \leq b_{n}\right) \\
\quad \leq E\left|a_{n i} X_{i}\right| \cdot \frac{\left|a_{n i} X_{i}\right|^{\alpha-1}}{b_{n}^{\alpha-1}} I\left(\left|X_{i}\right| \leq b_{n}\right) \\
\quad \leq b_{n}^{1-\alpha}\left|a_{n i}\right|^{\alpha} E\left|X_{i}\right|^{\alpha} I\left(\left|X_{i}\right| \leq b_{n}\right) \\
\quad \leq C b_{n}^{1-\alpha}\left|a_{n i}\right|^{\alpha} E|X|^{\alpha} I\left(|X| \leq b_{n}\right)+C b_{n}\left|a_{n i}\right|^{\alpha} P\left(|X|>b_{n}\right) \\
\leq C b_{n}^{1-\alpha}\left|a_{n i}\right|^{\alpha} E|X|^{\alpha} I\left(|X| \leq b_{n}\right)+C b_{n}^{1-\alpha}\left|a_{n i}\right|^{\alpha} E|X|^{\alpha} \\
\quad \leq C b_{n}^{1-\alpha}\left|a_{n i}\right|^{\alpha} E\left(\frac{|X|^{\alpha}}{(\log (1+|X|))^{\alpha / \gamma-1}} \cdot(\log (1+|X|))^{\alpha / \gamma-1}\right) \\
\quad \leq C n^{-1+1 / \alpha}(\log n)^{1 / \gamma-1}\left|a_{n i}\right|^{\alpha},
\end{aligned}
$$

we have

$$
\begin{aligned}
b_{n}^{-1} \sum_{i=1}^{n} E\left|a_{n i} X_{i}\right| I\left(\left|a_{n i} X_{i}\right|>b_{n},\left|X_{i}\right|>b_{n}\right) & \leq C b_{n}^{-1} n^{-1+1 / \alpha}(\log n)^{1 / \gamma-1} \sum_{i=1}^{n}\left|a_{n i}\right| \\
& \leq C(\log n)^{-1} \rightarrow 0, \quad \text { as } n \rightarrow \infty
\end{aligned}
$$

and

$$
\begin{aligned}
b_{n}^{-1} \sum_{i=1}^{n} E\left|a_{n i} X_{i}\right| I\left(\left|a_{n i} X_{i}\right|>b_{n},\left|X_{i}\right| \leq b_{n}\right) & \leq C b_{n}^{-1} n^{-1+1 / \alpha}(\log n)^{1 / \gamma-1} \sum_{i=1}^{n}\left|a_{n i}\right|^{\alpha} \\
& \leq C(\log n)^{-1} \rightarrow 0, \quad \text { as } n \rightarrow \infty .
\end{aligned}
$$


Thus, (3.29) holds by (3.30)-(3.34). Therefore, we only need to prove that

$$
\sum_{n=1}^{\infty} \frac{1}{n} P\left(\max _{1 \leq j \leq n}\left|\sum_{i=1}^{j}\left(Z_{i}-E Z_{i}\right)\right|>\varepsilon b_{n}\right)<\infty .
$$

Actually, by the Markov inequality, Lemma 2.2, Lemma 2.5, (3.18) and (3.28), we get

$$
\begin{aligned}
& \sum_{n=1}^{\infty} \frac{1}{n} P\left(\max _{1 \leq j \leq n}\left|\sum_{i=1}^{j}\left(Z_{i}-E Z_{i}\right)\right|>\varepsilon b_{n}\right) . \\
& \quad \leq C \sum_{n=1}^{\infty} n^{-1} b_{n}^{-2} \sum_{i=1}^{n} E\left(Z_{i}-E Z_{i}\right)^{2} \\
& \quad \leq C \sum_{n=1}^{\infty} n^{-1} b_{n}^{-2} \sum_{i=1}^{n} E\left|a_{n i} X\right|^{2} I\left(\left|a_{n i} X_{i}\right| \leq b_{n}\right) \\
& \quad \leq C \sum_{n=1}^{\infty} n^{-1} b_{n}^{-2} \sum_{i=1}^{n} E\left|a_{n i} X\right|^{2} I\left(\left|a_{n i} X\right| \leq b_{n}\right)+C \sum_{n=1}^{\infty} n^{-1} \sum_{i=1}^{n} P\left(\left|a_{n i} X\right|>b_{n}\right) \\
& \quad \leq C \sum_{n=1}^{\infty} n^{-1} b_{n}^{-2} \sum_{i=1}^{n} E\left(\left|a_{n i} X\right|^{\alpha} \cdot\left|a_{n i} X\right|^{2-\alpha}\right) I\left(\left|a_{n i} X\right| \leq b_{n}\right)+C \\
& \quad \leq C \sum_{n=1}^{\infty} n^{-1} b_{n}^{-\alpha} \sum_{i=1}^{n} E\left|a_{n i} X\right|^{\alpha} I\left(\left|a_{n i} X\right| \leq b_{n}\right)<\infty .
\end{aligned}
$$

Thus, the proof of Theorem 3.2 is completed.

\section{Conclusions}

In this paper, we use different methods from those of Sung [2] and Chen and Sung [3] to prove the results, and we obtain some strong convergence results for weighted sums of NSD random variables without the assumption of an identical distribution. Our results extend and improve the corresponding ones of Cai [1] and Sung [2] and Chen and Sung [3] to the case of NSD random variables.

\section{Acknowledgements}

The authors greatly appreciate both the Editor-in-Chief SH Sung and the referees for their valuable comments and some helpful suggestions that improved the clarity and readability of this paper. This research is supported by the National Natural Science Foundation of China (71271042; 11661029; 11661030).

\section{Competing interests}

The authors declare that they have no competing interests.

\section{Authors' contributions}

BM and DW carried out the design of the study and performed the analysis. QW participated in its design and coordination. All authors read and approved the final manuscript.

\section{Author details}

'School of Mathematical Science, University of Electronic Science and Technology of China, Chengdu, Sichuan 611731, P.R. China. ${ }^{2}$ College of Science, Guilin University of Technology, Guilin, Guangxi 541004, P.R. China.

\section{Publisher's Note}

Springer Nature remains neutral with regard to jurisdictional claims in published maps and institutional affiliations. 


\section{References}

1. Cai, GH: Strong laws for weighted sums of NA random variables. Metrika 68, 323-331 (2008)

2. Sung, SH: On the strong convergence for weighted sums of random variables. Stat. Pap. 52, 447-454 (2011)

3. Chen, PY, Sung, SH: On the strong convergence for weighted sums of negatively associated random variables. Stat. Probab. Lett. 92, 45-52 (2014)

4. Kemperman, JHB: On the FKG-inequalities for measures on a partially ordered space. Proc. K. Ned. Akad. Wet., Ser. A, Indag. Math. 80, 313-331 (1977)

5. Hu, TZ: Negatively superadditive dependence of random variables with applications. Chinese J. Appl. Probab. Statist. $16,133-144(2000)$

6. Joag-Dev, K, Proschan, F: Negative association of random variables with applications. Ann. Stat. 11(1), $286-295$ (1983)

7. Christofides, TC, Vaggelatou, E: A connection between supermodular ordering and positive/negative association. J. Multivar. Anal. 88, 138-151 (2004)

8. Eghbal, N, Amini, M, Bozorgnia, A: Some maximal inequalities for quadratic forms of negative superadditive dependence random variables. Stat. Probab. Lett. 80, 587-591 (2010)

9. Shen, Y, Wang, XJ, Yang, WZ, Hu, SH: Almost sure convergence theorem and strong stability for weighted sums of NSD random variables. Acta Math. Sin. Engl. Ser. 29(4), 743-756 (2013)

10. Shen, AT, Zhang, Y, Volodin, A: Applications of the Rosenthal-type inequality for negatively superadditive dependent random variables. Metrika 78, 295-311 (2015)

11. Wang, XJ, Deng, X, Zheng, LL, Hu, SH: Complete convergence for arrays of rowwise negatively superadditive dependent random variables and its applications. Statistics 48, 834-850 (2014)

12. Shen, Y, Wang, XJ, Hu, SH: On the strong convergence and some inequalities for negatively superadditive dependent sequences. J. Inequal. Appl. 2013, 448 (2013)

13. Wang, XJ, Shen, AT, Chen, ZY, Hu, SH: Complete convergence for weighted sums of NSD random variables and its application in the EV regression model. Test 24, 166-184 (2015)

14. Hsu, PL, Robbins, H: Complete convergence and the law of large numbers. Proc. Natl. Acad. Sci. USA 33(2), 25-31 (1947)

15. Erdös, P: On a theorem of Hsu and Robbins. Ann. Math. Stat. 20(2), 286-291 (1949)

16. Sung, SH: Complete convergence for weighted sums of random variables. Stat. Probab. Lett. 77, 303-311 (2007)

17. Huang, HW, Wang, DC, Wu, QY, Zhang, QX: A note on the complete convergence for sequences of pairwise NQD random variables. J. Inequal. Appl. 2011, 92 (2011)

18. Kuczmaszewska, A: On complete convergence in Marcinkiewicz-Zygmund type SLLN for negatively associated random variables. Acta Math. Hung. 128, 116 (2010)

19. Baek, J, Park, ST: Convergence of weighted sums for arrays of negatively dependent random variables and its applications. J. Stat. Plan. Inference 140, 2461-2469 (2010)

20. Gut, A: Complete convergence for arrays. Period. Math. Hung. 25, 51-75 (1992)

21. Li, W, Chen, PY, Sung, SH: Remark on convergence rate for weighted sums of $\rho^{*}$-mixing random variables. Rev. R. Acad. Cienc. Exactas Fís. Nat., Ser. A Mat. 111, 507-513 (2017)

22. Sung, $\mathrm{SH}$ : On the strong convergence for weighted sums of $\rho^{*}$-mixing random variables. Stat. Pap. 54, 773-781 (2013)

23. Wu, QY: Complete convergence for weighted sums of sequences of negatively dependent random variables. J. Probab. Stat. 2011, Article ID 202015 (2011)

\section{Submit your manuscript to a SpringerOpen ${ }^{\circ}$ journal and benefit from:}

- Convenient online submission

- Rigorous peer review

- Open access: articles freely available online

- High visibility within the field

- Retaining the copyright to your article

Submit your next manuscript at $\boldsymbol{s p r i n g e r o p e n . c o m ~}$ 